\title{
The relationship between pain and mood following spinal cord injury
}

\author{
Paul Kennedy ${ }^{1,2}$, Laurence Hasson ${ }^{1}$ \\ ${ }^{1}$ Department of Clinical Psychology, National Spinal Injuries Centre, Stoke Mandeville Hospital, \\ Buckinghamshire, UK, ${ }^{2}$ Oxford Institute of Clinical Psychology Training, University of Oxford, UK
}

Objectives: To explore the relationship between pain and mood during spinal cord injury rehabilitation, and to discuss clinical implications to optimize rehabilitation outcomes.

Design: Repeated measures, retrospective cohort study.

Setting: Tertiary care, spinal cord injury rehabilitation center.

Participants: Patients ( $N=509$ ) who completed both Needs Assessment Checklist (NAC) 1 and NAC2 between February 2008 and February 2015.

Interventions: Not applicable.

Outcome measure: Pain ratings (0-10) and mood scores (0-24) were obtained from the Needs Assessment Checklist (NAC). NAC1 is completed within 4 weeks post-mobilization and NAC2 upon the patient moving to the pre-discharge ward.

Results: There were statistically significant improvements in both pain and mood from NAC1 to NAC2. There were significant correlations between pain and mood at both NAC1 and NAC2 (a decrease in pain was associated with an improvement in mood). Individuals who reported that pain interfered with their rehabilitation had higher pain scores and lower mood scores at both NAC1 and NAC2.

Conclusions: Pain and mood evidently interact following spinal cord injury, and the nature of this relationship is complex. The current study provides some support for the bidirectional causality hypothesis, suggesting that pain and mood exert an effect upon each other. It is important to address pain and psychological issues early and together in the post-injury phase to optimize rehabilitation outcomes.

Keywords: Depression, Mood, Pain, Rehabilitation, Spinal cord injury

\section{Introduction}

Spinal cord injury (SCI) can have a devastating and typically long-term impact on a person's physical, functional and psychological domains, and pain is a pervasive and recurrent issue which can often have a debilitating effect for many people following SCI. While reported prevalence rates vary dramatically, ${ }^{1}$ it is estimated that around $65 \%$ of individuals with SCI suffer from chronic pain, ${ }^{2,3}$ with more than half categorizing their pain as "severe" or "excruciating." 3 Pain has been shown to have a significant impact on people's daily life beyond the functional consequences of their $\mathrm{SCI},{ }^{4}$ and people with pain experience a lower quality of life. ${ }^{5}$ Research has also found that the prevalence of

Correspondence to: Paul Kennedy, Department of Clinical Psychology, The National Spinal Injuries Centre, Stoke Mandeville Hospital, Mandeville Road, Aylesbury, Buckinghamshire, HP21 8AL, UK. Email: paul.kennedy@ hmc.ox.ac.uk. pain was greater 5 years post-injury than at 6 months, which highlights the often persistent nature of pain following SCI and emphasizes the importance of treating pain in the long-term. ${ }^{3}$ Furthermore, studies suggest that there are few effective pharmacological treatments available for chronic pain following SCI. ${ }^{6}$ This is particularly true for neuropathic pain which is often experienced post-SCI, and whilst some medications are effective at treating nociceptive pain, they often have limited usefulness against neuropathic pain. ${ }^{7,8}$

The psychological impact of spinal cord injury is well documented. Research suggests that approximately onequarter to one-third of people with SCI will experience significant levels of depression following their injury. ${ }^{9}$ Furthermore, longitudinal studies of the psychological impact of SCI have found that depression is highly prevalent for many years post-discharge, and depression is also associated with a higher incidence of secondary 
health complications, such as pressure sores and urinary tract infections. ${ }^{9,10}$ Whilst the majority of individuals with SCI exhibit largely stable psychological resilience, a number of distinct mental health trajectories illustrate the highly diverse nature of psychological impact following SCI, and emphasize the need to address psychological issues both during rehabilitation and postdischarge. ${ }^{11,12}$ Suicide rates among individuals with SCI remain more than three times higher than that of the general population, further highlighting the importance of psychological interventions throughout the lifespan of those with spinal cord injuries. ${ }^{13}$

The relationship between pain and mood following SCI is also well established, although the nature of this relationship is complex. Craig et al. ${ }^{14}$ found pain to be one of the most significant predictors of depression two years post-injury, and recommended pain management as an intervention to reduce depression following SCI. Numerous other studies have also found a significant relationship between pain and mood following SCI. ${ }^{2,4}$ Cairns et al. ${ }^{15}$ found that reducing pain affects depression more than reducing depression affects pain, providing support for the "linear causality model." Recent research has found chronic pain to contribute significantly to increased depressive mood following SCI, such that individuals with high pain were 8 times more likely to have a depressive mood. ${ }^{16}$ Pain intensity is also considered to be a significant predictor of depressed mood following SCI. ${ }^{17}$ Other research has suggested that pain and mood interact with each other, and that each exerts an impact on the other. ${ }^{18}$ Depression may make one more sensitive to pain, lower pain thresholds, intensify pain, or cause one to focus more on their pain. ${ }^{19,20}$ This in turn makes pain worse, which may make one more depressed-a vicious cycle, further mediated by the shared neurophysiological and neurochemical pathways common to both depression and pain. ${ }^{20}$

The biopsychosocial model is the most heuristic approach to chronic pain, and can be helpfully applied to spinal cord injury. This model takes into account not only the organic biological processes underlying pain, but also the psychological and social factors, and the interaction between these three domains. For example, catastrophizing is a negative psychological strategy which is associated with increased pain intensity, increased psychological distress and poorer response to pain treatments, as well as a lack of perceived social support. ${ }^{21,22}$ The biopsychosocial model is congruent with the idea that pain and mood each exert an impact on the other; that there is an interaction between the physical and psychological factors inherent in pain. The authors of the current study put forth a bidirectional causality model of pain and depression following SCI, which suggests that there exists a complex interaction between pain and psychological impact post-injury. This paper aims to explore the relationship between pain and mood during SCI rehabilitation, and it is hypothesized that the results will provide support for the bidirectional causality model.

\section{Methods}

\section{Participants}

All inpatients of the spinal injuries center routinely complete the Needs Assessment Checklist (NAC). This audit retrospectively examined all individuals who had completed both NAC1 and NAC2 between February 2008 and February 2015. Data were obtained for 509 patients. Participant demographics can be seen in Table 1.

\section{Materials and procedure}

The Needs Assessment Checklist is a comprehensive rehabilitation outcome measure designed specifically for individuals with spinal cord injuries, which has demonstrated good reliability and validity. ${ }^{23,24}$ Within the NAC, patients are asked to rate their pain on a scale of $0-10$, where 0 is "No pain" and 10 is "As bad as you can imagine.' The NAC also contains a mood questionnaire, which comprises 8 questions on a 4point scale $(0,1,2,3)$, giving a minimum score of 0 and a maximum score of 24 (higher scores mean better

\section{Table 1 Participant demographics}

\begin{tabular}{ll}
\hline Sex & \\
Male & $373(73.3 \%)$ \\
Female & $136(26.7 \%)$ \\
Age at NAC 1 & \\
Mean (SD) & $47.6(17.8)$ \\
Range & $15-88$ \\
Age Groups & \\
<30 & $107(21.3 \%)$ \\
$30-45$ & $120(23.9 \%)$ \\
$45-60$ & $128(25.5 \%)$ \\
$>60$ & $147(29.3 \%)$ \\
Missing & 7 \\
Severity of Injury & \\
C1-4 AIS A-C & $68(14.6 \%)$ \\
C5-8 AIS A-C & $76(16.3 \%)$ \\
T1-S5 AIS A-C & $218(46.7)$ \\
AIS D & $105(22.5 \%)$ \\
Missing & 42 \\
Cause of Injury & \\
Motor vehicle collision & $134(26.3 \%)$ \\
Fall & $129(25.3 \%)$ \\
Sports injury & $46(9.0 \%)$ \\
Non-traumatic & $179(35.2 \%)$ \\
Assault & $11(2.2 \%)$ \\
Other & $10(2.0 \%)$ \\
\hline
\end{tabular}


mood). When interpreting the score to this measure, those who score below 38\% would be considered as potential psychological cases. Previous research has shown that the NAC's measure of psychological impact has significant concurrent validity with the depression scale of the Hospital Anxiety and Depression Scale (HADS). ${ }^{24}$ NAC1 is completed within 4 weeks post-mobilization, mobilization is classified as when a patient is able to sit up in their chair for 4 hours. The NAC2 is completed when the patient is moved onto the pre-discharge ward, which is usually approximately 6 weeks before they are discharged. Between these times, patients have regular goal planning meetings with their multidisciplinary team, to set targets which structure their rehabilitation. All data are entered into a database and stored in accordance with Trust confidentiality guidelines.

\section{Results}

A paired samples $t$-test showed a statistically significant (though not clinically meaningful) reduction in pain rating from $\mathrm{NAC} 1($ mean $=3.60)$ to $\mathrm{NAC} 2($ mean $=$ $3.34), t(508)=2.055, \mathrm{P}=0.04$. Pain rating at $\mathrm{NAC} 1$ significantly correlated with pain rating at NAC2, $r=$ $0.433, \mathrm{~N}=509, \mathrm{P}<0.001$.

A paired samples $t$-test showed some improvement in mood from NAC1 $($ mean $=18.58)$ to NAC2 $($ mean $=$ 19.17), $t(504)=-3.35, \mathrm{P}=0.001$. Mood at NAC1 significantly correlated with mood at NAC2, $r=0.531$, $\mathrm{N}=505, \mathrm{P}<0.001$.

\section{Relationship between pain and mood}

There was a significant correlation between pain and mood at both NAC1 and NAC2 (Table 2). In all cases higher pain is significantly associated with lower mood.

There was a significant correlation between change in pain and change in mood from NAC1 to NAC2, $r=$ $-0.130, \mathrm{~N}=505, \mathrm{P}=0.004$, meaning a reduction in pain is associated with an improvement in mood (note the correlation is negative because a higher score on the pain scale means worse pain, and a higher score on the mood scale means better mood).

\begin{tabular}{|c|c|c|c|}
\hline$N=506$ & & Mood NAC1 & Mood NAC2 \\
\hline $\begin{array}{c}\text { Pain rating } \\
\text { NAC1 }\end{array}$ & $\begin{array}{l}\text { Pearson } \\
\quad \text { Correlation } \\
\text { Sig. (2-tailed) }\end{array}$ & $\begin{array}{l}-0.289 \\
<0.001\end{array}$ & $\begin{array}{l}-0.225 \\
<0.001\end{array}$ \\
\hline \multirow[t]{2}{*}{$\begin{array}{c}\text { Pain rating } \\
\text { NAC2 }\end{array}$} & $\begin{array}{l}\text { Pearson } \\
\text { Correlation } \\
\text { Sia (2-tailed) }\end{array}$ & -0.242 & -0.310 \\
\hline & Sig. (2-tailed) & $<0.001$ & $<0.001$ \\
\hline
\end{tabular}

Table 3 Mean pain rating at NAC1 and NAC2 by Pain Interference group

\begin{tabular}{lccccc}
\hline & \multicolumn{2}{c}{ NAC 1 } & & & NAC 2 \\
\cline { 2 - 3 } \cline { 5 - 6 } $\begin{array}{l}\text { Does pain interfere with } \\
\text { your rehabilitation? }\end{array}$ & $\mathrm{N}$ & $\begin{array}{c}\text { Mean pain } \\
\text { rating }\end{array}$ & & $\mathrm{N}$ & $\begin{array}{c}\text { Mean pain } \\
\text { rating }\end{array}$ \\
\hline No & 304 & 2.52 & & 337 & 2.39 \\
Yes & 202 & 5.21 & & 171 & 5.23 \\
\hline
\end{tabular}

Table 4 Mean mood rating at NAC1 and NAC2 by Pain Interference group

\begin{tabular}{lcc}
\hline $\begin{array}{l}\text { Does pain interfere with } \\
\text { your rehabilitation? }\end{array}$ & $\begin{array}{c}\text { Mean mood } \\
\text { rating at NAC 1 }\end{array}$ & $\begin{array}{c}\text { Mean mood } \\
\text { rating at NAC 2 }\end{array}$ \\
\hline No & 19.49 & 20.02 \\
Yes & 17.25 & 17.53 \\
\hline
\end{tabular}

Pain interference with rehabilitation

Patients were asked the question: "Does pain interfere with your ability to get on with your rehabilitation?" Results can be seen in Table 3. A McNemar test showed a significant difference between the proportion of "Yes" and "No" responses from NAC1 to NAC2, $\chi^{2}(\mathrm{~N}=505)=6.78, \mathrm{P}=0.009$. Pain ratings differ significantly between the groups at both NAC1 $(t(504)=$ $-13.17, \mathrm{P}<0.001)$ and NAC2 $(t(506)=-12.63, \mathrm{P}<$ $0.001)$.

There is also some indication that mood ratings differ between the pain interference groups at both NAC1 $(t(501)=6.498, \mathrm{P}<0.001)$ and NAC2 $(t(505)=6.52$, $\mathrm{P}<0.001$ ) (see Table 4), such that patients whose pain does interfere with their rehabilitation have lower mood than those whose pain does not interfere with their rehabilitation.

\section{Discussion}

The correlation between change in pain and change in mood from NAC1 to NAC2 provides some support of previous findings, that there exists an important association between pain and psychological impact following SCI. ${ }^{14-18}$ A reduction in pain over the course of rehabilitation is related to an improvement in mood. Furthermore, those patients who report that pain interferes with their rehabilitation also report lower mood at both NAC1 and NAC2, demonstrating the real consequences that pain and psychological impact can have on a patient's recovery. The correlations between pain at $\mathrm{NAC} 1$ and mood at NAC2, and between mood at $\mathrm{NACl} 1$ and pain at NAC2 provide some support to the bidirectional causality hypothesis, that both pain and mood exert an effect upon each other, and this can 
result in a downward spiral of pain and depression in some cases, with each factor having a negative impact on the other (a vicious cycle).

The results provide further support for the efficacy of the needs assessment checklist as a method of assessing general rehabilitation outcomes including pain and mood during spinal cord injury rehabilitation.

The complex interaction of pain and psychological impact following SCI has a number of clinical implications for the rehabilitation process. Research has found that almost all patients report experiencing pain during rehabilitation. ${ }^{25}$ It is important to address both pain and mood early in the post-injury phase, to reduce their impact on rehabilitation and to optimize outcomes, and therefore assessment tools should be utilized early and often to diagnose potential issues. A combination of psychological and pharmacological interventions to treat both pain and depression may be beneficial in helping patients who suffer from pain and low mood. Psychological interventions are especially relevant for treating neuropathic pain which is poorly managed by medication. ${ }^{6,7}$ Siddall and Middleton ${ }^{26}$ proposed an algorithm for diagnosing and managing pain following SCI which incorporates medication, psychological intervention and a variety of other factors, and clinicians may benefit from utilizing this system. A cognitive behavioral pain management program for people with spinal cord injury has also been found to increase quality of life, ${ }^{27}$ and there is comprehensive evidence to support the effectiveness of CBT for managing co-morbid depression following $\mathrm{SCI},{ }^{28}$ further highlighting the importance and efficacy of psychological interventions for both pain and mood. It is critical that pain following SCI is approached and treated in the context of the biopsychosocial model, and that both pain and depression are treated early and together to optimize rehabilitation outcomes.

\section{Study limitations}

A limitation of the current study is that it only assesses one aspect of pain, and there are evidently other factors involved which this study has not explored. There is a need for further research to examine what other factors are involved and to further explore this complex relationship.

Future research may wish to further analyze the relationship between pain and mood, starting earlier post-injury and utilizing more time points and a variety of different pain and mood measures, to clarify the nature of this relationship and how it develops longitudinally. Further studies may examine the effectiveness of psychological and pharmacological interventions in treating the combination of pain and depression after SCI, and may wish to explore the shared neurophysiological and neurochemical pathways of pain and mood.

\section{Conclusions}

Pain and mood evidently interact following spinal cord injury, and the nature of this relationship is complex. The current study suggests that bidirectional causality is the most likely explanation of this relationship. It is important to address pain and psychological issues early and together in the post-injury phase to optimize rehabilitation outcomes. Further research is needed to elucidate this complex relationship.

\section{Acknowledgments}

We thank all patients and staff at the National Spinal Injuries Centre, Stoke Mandeville Hospital, who participated in this study. We also thank Lydia Garmon-Jones, Assistant Psychologist, National Spinal Injuries Centre, Stoke Mandeville Hospital, for assisting with manuscript preparation.

\section{Disclaimer statements \\ Contributors None.}

\section{Funding None.}

Conflict of interest The authors declare no conflict of interest.

Ethics approval All local research and ethics procedures were complied with.

\section{References}

1 Dijkers M, Bryce T, Zanca J. Prevalence of chronic pain after traumatic spinal cord injury: a systematic review. J Rehabil Res Dev 2009;46(1):13-29.

2 Nicholson Perry K, Nicholas MK, Middleton J. Spinal cord injury-related pain in rehabilitation: a cross-sectional study of relationships with cognitions, mood and physical function. Eur J Pain 2009;13(5):511-7.

3 Siddall PJ, McClelland JM, Rutkowski SB, Cousins MJ. A longitudinal study of the prevalence and characteristics of pain in the first 5 years following spinal cord injury. Pain 2003;103(3): 249-57.

4 Raichle KA, Hanley M, Jensen MP, Cardenas DD. Cognitions, coping and social environment predict adjustment to pain in spinal cord injury. J Pain 2007;8(9):718-29.

5 Barrett H, McClelland JM, Rutkowski SB, Siddall PJ. Pain characteristics in patients admitted to hospital with complications after spinal cord injury. Arch Phys Med Rehabil 2003;84(6):789-95.

6 Warms CA, Turner JA, Marshall HM, Cardenas DD. Treatments for chronic pain associated with spinal cord injuries: many are tried, few are helpful. Clin J Pain 2002;18(3):154-63.

7 Richards JS, Bombardier CH, Wilson CS, Chiodo AE, Brooks L, Tate DG, et al. Efficacy of Venlafaxine XR for the treatment of pain in patients with spinal cord injury and major depression: a randomized, controlled trial. Arch Phys Med Rehabil 2015;96(4): 680-9.

8 Guy S, Mehta S, Leff L, Teasell R, Loh E. Anticonvulsant medication use for the management of pain following spinal cord 
injury: systematic review and effectiveness analysis. Spinal Cord 2014;52(2):89-96.

9 Pollard C, Kennedy P. A longitudinal analysis of emotional impact, coping strategies and post-traumatic psychological growth following spinal cord injury: a 10-year review. $\mathrm{Br} \mathrm{J}$ Health Psychol 2007;12(Pt 2):347-62.

10 Hoffman JM, Bombardier CH, Graves DE, Kalpakjian CZ, Krause JS. A longitudinal study of depression from 1 to 5 years after spinal cord injury. Arch Phys Med Rehabil 2011;92(3):411-8.

11 Bonnano GA, Kennedy P, Galatzer-Levy IR, Lude P, Elfström ML. Trajectories of resilience, depression, and anxiety following spinal cord injury. Rehabil Psychol 2013;57(3):236-247.

12 van Leeuwen CM, Hoekstra T, van Koppenhagen CF, de Groot S, Post MW. Trajectories and predictors of the course of mental health after spinal cord injury. Arch Phys Med Rehabil 2012; 93(12):2170-6.

13 Cao Y, Massaro JF, Krause JS, Chen Y, Devivo MJ. Suicide mortality after spinal cord injury in the United States: injury cohorts analysis. Arch Phys Med Rehabil 2014;95(2):230-5.

14 Craig AR, Hancock KM, Dickson HG. Spinal cord injury: a search for determinants of depression two years after the event. Br J Clin Psychol 1994;33(Pt 2):221-30.

15 Cairns DM, Adkins RH, Scott MD. Pain and depression in acute traumatic spinal cord injury: origins of chronic problematic pain? Arch Phys Med Rehabil 1996;77(4):329-35.

16 Craig A, Tran Y, Siddall P, Wijesuriya N, Lovas J, Bartrop R et al. Developing a model of associations between chronic pain, depressive mood, chronic fatigue, and self-efficacy in people with spinal cord injury. J Pain 2013;14(9):911-20.

17 Craig A, Rodrigues D, Tran Y, Guest R, Bartrop R, Middleton J. Developing an algorithm capable of discriminating depressed mood in people with spinal cord injury. Spinal Cord 2014;52(5): 413-6.

18 Rintala D, Loubser PG, Castro J, Hart KA, Fuhrer MJ. Chronic pain in a community-based sample of men with spinal cord injury: prevalence, severity, and relationship with impairment, disability, handicap, and subjective well-being. Arch Phys Med Rehabil 1998;79(6):604-614.

19 Bair MJ, Robinson RL, Katon W, Kroenke K. Depression and pain comorbidity: a literature review. Arch Intern Med 2003; 163(20):2433-45.

20 Robinson MJ, Edwards SE, Iyengar S, Bymaster F, Clark M, Katon W. Depression and pain. Front Biosci (Landmark Ed) 2009;14:5031-51.

21 Hanley MA, Raichle K, Jensen M, Cardenas DD. Pain catastrophizing and beliefs predict changes in pain interference and psychological functioning in persons with spinal cord injury. $\mathbf{J}$ Pain 2008;9(9):863-71.

22 Turner JA, Jensen MP, Warms CA, Cardenas DD. Catastrophizing is associated with pain intensity, psychological distress, and pain-related disability among individuals with chronic pain after spinal cord injury. Pain 2002;98(1-2):127-34.

23 Kennedy P, Hamilton LR. The needs assessment checklist: a clinical approach to measuring outcome. Spinal Cord 1999;37(2): 136-9.

24 Berry C, Kennedy P. A psychometric analysis of the Needs Assessment Checklist (NAC). Spinal Cord 2003;41(9):490-501.

25 New PW, L. T. (1997). A survey of pain during rehabilitation after acute spinal cord injury. Spinal Cord 1997;35(10):658-63.

26 Siddall PJ, Middleton JW. A proposed algorithm for the management of pain following spinal cord injury. Spinal Cord 2006;44(2): $67-77$.

27 Perry KN, Nicholas MK, Middleton J. Multidisciplinary cognitive behavioural pain management programmes for people with a spinal cord injury: design and implementation. Disabil Rehabil 2011;33(13-14):1272-80.

28 Perkes SP, Bowman J, Penkala S. Psychological therapies for the management of co-morbid depression following a spinal cord injury: a systematic review. J Health Psychol 2014;19(12): $1597-612$. 